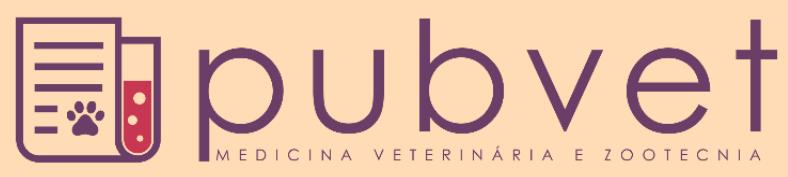

https://doi.org/10.31533/pubvet.v12n12a222.1-7

\title{
Ultrassonografia abdominal como método complementar no estadiamento do linfoma canino
}

\author{
Felipe Foletto Geller ${ }^{1}$, Carmel Rezende Dadalto ${ }^{2 *} \bullet$, Letícia Rocha Inamassu ${ }^{3}$, Danuta Pulz \\ Doiche $^{4}$, Shayra Peruchi Bonatelli ${ }^{5}$, Maria Jaqueline Mamprim ${ }^{6} \bullet$ \\ ${ }^{1}$ Professor Doutor da Universidade Federal de Mato Grosso do Sul, Campo Grande-MS, Brasil. E-mail: \\ ${ }^{2}$ Doutoranda no Programa de Pós-Graduação em Biotecnologia Animal, Universidade Estadual Paulista, campus Botucatu-SP. \\ ${ }^{3}$ Doutoranda no Programa de Pós-Graduação em Animais Silvestres, Universidade Estadual Paulista, campus Botucatu-SP. \\ ${ }^{4}$ Médica veterinária autônoma. Botucatu-SP Brasil. \\ ${ }^{5}$ Doutoranda no Programa de Pós-Graduação em Biotecnologia Animal, Universidade Estadual Paulista, campus Botucatu-SP. \\ ${ }^{6}$ Professora da Universidade Estadual Paulista, Departamento de Radiologia Veterinária. Botucatu-SP Brasil. \\ * Autor para correspondência, E-mail: carmel_dadalto@outlook.com
}

\begin{abstract}
RESUMO. O linfoma canino é uma das neoplasias mais frequentes encontradas na medicina veterinária e pode acometer vários órgãos. $\mathrm{O}$ exame ultrassonográfico tem demonstrado grande sensibilidade em detectar mudanças parenquimatosas mesmo antes que os exames laboratoriais estejam alterados; além de ser considerado como um importante advento para auxiliar no estadiamento de doenças e guiar colheita de material. O objetivo desse trabalho foi pesquisar a contribuição do exame ultrassonográfico no linfoma canino de 70 animais e propor um estadiamento entre estágios de I a V das lesões abdominais em cães com diagnóstico citológico ou histopatológico de linfoma. Nesse estudo foi possível por meio a da classificação clínica da Organização Mundial da Saúde (OMS) modificada, determinar em estágio I 28,6 \% dos animais, $1,4 \%$ estágio II, $41,4 \%$ no estágio IV e $28,6 \%$ estágio V e não foram observados animais no estágio III. A despeito da grande variação do aspecto ultrassonográfico dessas lesões nos órgãos linfoides, o acometimento dos linfonodos ilíacos mediais foi estatisticamente e demonstrou ser um importante sinal sentinela da presença do linfoma nesses animais. Portanto, a imagem ultrassonográfica abdominal é de extrema relevância na rotina clínica, pois possibilita o estadiamento do linfoma abdominal, seu prognóstico, bem como monitora terapêutica instituída.
\end{abstract}

Palavras chave: diagnóstico por imagem, ilíaco medial, linfonodos, neoplasia

\section{Abdominal ultrasound as a complementary method for staging canine lymphoma}

ABSTRACT. Canine lymphoma is one of the most frequent neoplasms in veterinary
medicine and it can affect several organs. Ultrasound examination has shown great
sensitivity in detecting parenchymal abnormalities even before laboratory tests change, and
it is considered an important advent to help staging diseases and to guide material
collection. The main objective of this study was to investigate the contribution of
ultrasonographic examination canine lymphoma in 70 animals and to propose staging
between stages I to V, based on abdominal lesions of dogs with cytological or
histopathological diagnosis of lymphoma. In this study, through a modified World Health
Organization clinical classification, it could be determined that $28.6 \%$ of the animals were
within stage I, $1.4 \%$ stage II, $41.4 \%$ in stage IV and $28.6 \%$ stage $\mathrm{V}$ and no stage III animals
were observed. Despite the great variation of the ultrasonographic appearance of these
lesions in lymphoid organs, the involvement of medial iliac lymph nodes was statistically
significant and proved to be an important sentinel sign of the presence of lymphoma in 
these animals. Therefore, abdominal ultrasound imaging is of extreme relevance in clinical routine, as it allows staging abdominal lymphoma, its prognosis, as well as monitoring the treatment stablished.

Keywords: imaging, medial iliac, lymph nodes, cancer

\section{Ecografía abdominal como método complementario en la estadificación de linfoma canino}

RESUMEN. El linfoma canino es una neoplasia frecuentemente encontrada en la medicina veterinaria y puede afectar varios órganos. El examen ecográfico ha demostrado gran sensibilidad en detectar cambios parenquimatosos antes de que los exámenes de laboratorio estén alterados, además de ser considerado como un importante advenimiento para auxiliar en la estadificación de enfermedades y guiar la recolección de material. El principal objetivo de este trabajo fue investigar la contribución del examen ecográfico en linfoma canino de 70 animales y proponer una estadificación del entre etapas de I a V de las lesiones abdominales en perros con diagnóstico citológico o histopatológico de linfoma. En este estudio fue posible por medio de la clasificación clínica de la Organización Mundial de la Salud (OMS) modificada, determinar en estadio I $28,6 \%$ de los animales, $1,4 \%$ estadio II, $41,4 \%$ en la etapa IV y $28,6 \%$ de la etapa V y no se observaron animales en la etapa III. A pesar de la gran variación del aspecto ecográfico de estas lesiones en los órganos linfoides, el acometimiento de los ganglios linfáticos ilíacos mediáticos fue estadísticamente significativo y demostró ser un importante signo centinela de la presencia del linfoma en esos animales. Por lo tanto, la imagen ecográfica abdominal es de extrema relevancia en la rutina clínica, pues posibilita la estadificación del linfoma abdominal, su pronóstico, así como monitora terapéutica instituida.

Palabras clave: diagnóstico por imagen, ilíaco medial, ganglios linfáticos, neoplasia

\section{Introdução}

O linfoma é uma neoplasia hematopoiética que se origina das células linfoides de órgãos como baço, fígado ou linfonodos. A forma multicêntrica é a de maior ocorrência (Vail 2000; Jacobs et al. 2002). O estadiamento clínico do linfoma canino envolve determinar locais de acometimento baseado em critérios da OMS, que classifica a extensão da doença, importante para o prognóstico (Crabtree et al. 2010). O diagnóstico definitivo é realizado por meio da citologia aspirativa por agulha fina (CAAF) ou biópsia de órgãos acometidos e linfonodos (Vail \& Young 2007). Devendo ressaltar que é preferível à punção de linfonodos poplíteos e pré-escapulares, evitando linfonodos que drenam áreas reativas como os mandibulares (Dickinson 2008). O exame ultrassonográfico de pacientes oncológicos é realizado para auxiliar a localização de lesões e guiar citologias ou biópsias, reduzindo procedimentos mais invasivos (Wisner \& Pollard 2004; Nyman et al. 2005). As alterações ultrassonográficas associadas ao linfoma são variadas, em fígado pode apresentar nódulos hipoecogênicos, lesões em alvo ou alteração de ecogenicidade difusa do parênquima, no baço as alterações são difusas hipoecogênicas ou de ecogênicidade mista, lesões em alvo ou nódulos pequenos, porém esses padrões não são patognomônicos da doenças (Nyman et al. 2005; Crabtree et al. 2010). Os linfonodos que apresentam infiltrados neoplásicos aumentam de tamanho e se tornam hipoecogênicos, podendo apresentar margens irregulares, espessamentos corticais, ausência de hilo e aumento da relação entre eixo curto/longo (Llabrés-Díaz 2004).

Esse trabalho teve como objetivo analisar as alterações ultrassonográficas abdominais em cães com diagnóstico de linfoma canino, a fim de propor o estadiamento ultrassonográfico de I a V da doença em cães, baseado no sistema de estadiamento clínico da OMS do linfoma em animais domésticos, visando nortear o clínico quanto ao prognóstico e monitoramento do tratamento oncológico.

\section{Material e métodos}

Foi realizado estudo retrospectivo de exames ultrassonográficos abdominais de 70 cães com 
diagnóstico de linfoma admitido no setor de diagnóstico por imagem da Faculdade de Medicina Veterinária e Zootecnia UNESP Botucatu-SP.

O critério de inclusão foi baseado no diagnóstico citológico ou histológico de linfoma em linfonodos superficiais ou de órgãos linfoides, realizados por patologistas experientes. E foram excluídos os pacientes com outros processos neoplásicos concomitantes ou afetados por outras doenças que poderiam causar alterações nas imagens ultrassonográficas.

Neste estudo as imagens ultrassonográficas foram revisadas por um único observador experiente para classificá-las baseado no sistema de estadiamento clínico (Quadro 1) da OMS para linfoma em animais domésticos, de forma adaptada para os achados ultrassonográficos. Foram considerados linfonodos alterados aqueles que obtiveram relação entre eixo curto/eixo longo superior a 0,7 como proposto por Llabrés-Díaz (2004).

Quadro 1. Sistema de estadiamento Clínico da OMS para o linfoma em animais domésticos, adaptado para o exame ultrassonográfico.

\begin{tabular}{|c|l|}
\hline Estágio & \multicolumn{1}{|c|}{ Parâmetros } \\
\hline I & $\begin{array}{l}\text { Um único linfonodo abdominal acometido ou um } \\
\text { único órgão linfoide. } \\
\text { II }\end{array}$ \\
III & $\begin{array}{l}\text { Linfonodos abdominais alterados de uma mesma } \\
\text { região anatômica. } \\
\text { Linfonodos abdominais alterados de diferentes } \\
\text { regiões anatômicas. } \\
\text { Alteração em fígado e/ou baço com acometimento } \\
\text { de linfonodos. } \\
\text { Alteração em fígado e/ou baço com acometimento } \\
\text { de linfonodos e outros órgãos abdominais }\end{array}$ \\
\hline
\end{tabular}
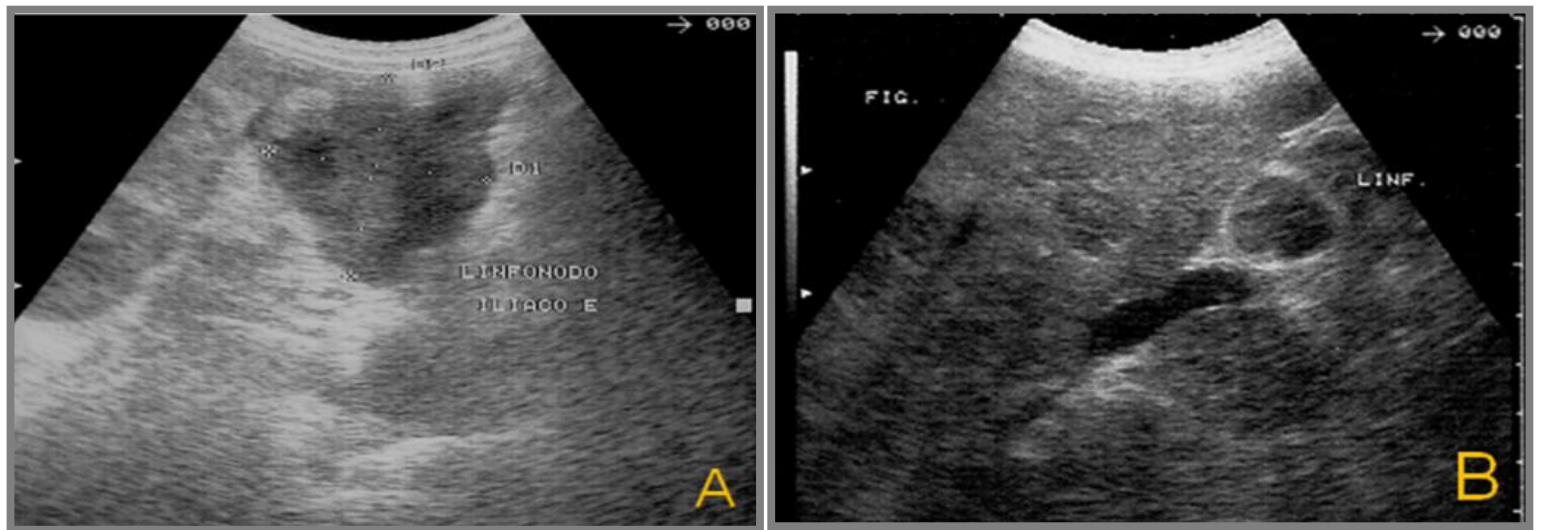

Figura 1. Imagem ultrassonográfica de aumento dos linfonodos abdominais no mesmo animal. A) Linfonodo Ilíaco Medial Esquerdo B) Linfonodo Hepático.
As imagens avaliadas foram obtidas utilizando equipamento de ultrassom: GE duplex scan com transdutores multifrequenciais, convexo de 3,5 a $5 \mathrm{MHz}$ e linear de 6 a $10 \mathrm{MHz}$.

O estudo da distribuição das incidências foi realizado considerando o teste Qui-quadrado para uma amostra e o nível de $5 \%$ de significância.

\section{Resultados}

$\mathrm{Na}$ avaliação ultrassonográfica abdominal dos 70 cães, $84,3 \%$ apresentaram alteração do parênquima hepático e $78,6 \%$ do esplênico. A hepatomegalia foi identificada em 37 animais $(52,9 \%)$, esplenomegalia em 27 animais $(38,6 \%)$ e linfadenomegalia em 37 animais $(57,1 \%)$.

Dos 37 cães que apresentaram linfadenomegalia abdominal a maior ocorrência foi aumento dos linfonodos ilíacos mediais em 23 animais $(62,1 \%)$ e em menor número os linfonodos hepáticos, esplênicos, lombar aórtico, mesentérico cranial, renal e gástrico (Tabela 1). Alguns animais apresentaram mais de um linfonodo aumentado (Figura 1).

Tabela 1. Avaliação ultrassonográfica de linfadenomegalia nos animais acometidos por linfoma.

\begin{tabular}{lr}
\hline Linfonodos & Animais \\
\hline Ilíaco medial & $23 \mathrm{a}$ \\
Hepáticos & $11 \mathrm{~b}$ \\
Esplênicos & $11 \mathrm{~b}$ \\
Lombar aórtico & $10 \mathrm{~b}$ \\
Mesentérico cranial & $7 \mathrm{~b}$ \\
Renalo & $6 \mathrm{~b}$ \\
Gástrico & \\
\hline Médias seguidas pela mesma letra não & diferem \\
estatisticamente entre si (P > 0,05). & \\
\end{tabular}


Da amostragem deste estudo, 59 animais possuíam exames com alterações ultrassonográficas hepáticas, sendo que 53 $(89,8 \%)$ apresentaram alterações difusas do parênquima, diferindo estatisticamente de outras alterações (Tabela 2). Apenas cinco dos 59 animais tiveram o linfoma como diagnóstico definitivo pela punção hepática. Dois deles apresentavam hepatomegalia com alteração difusa da ecogenicidade do parênquima, um com hiperecogênicidade hepática e outro hipoecogênicidade. Os outros três cães exibiram lesões focais e bem definidas em parênquima, dos quais dois com lesões mistas e dimensões hepáticas preservadas e um com lesão do tipo alvo, e tamanho hepático preservado (Figura 2).

Tabela 2. Aspectos ultrassonográficos hepáticos sugestivos de linfoma observados em cães com diagnóstico de linfoma por citologia de linfonodo superficial.

\begin{tabular}{lccccc}
\hline Alterações do Parênquima & \multicolumn{2}{c}{ Animais } & \multicolumn{2}{c}{ Hepatomegalia } \\
Hepático & $\mathrm{n}$ & $\%$ & Presença & $\%$ \\
\hline Focais & & & & \\
Lesões hipoecogênicas & $05 \mathrm{~b}$ & 8,5 & $03 \mathrm{~b}$ & 8,1 \\
Lesão tipo alvo & $01 \mathrm{~b}$ & 1,7 & $0 \mathrm{~b}$ & 0,0 \\
Difusas & & & & \\
Hipoecogênicidade & $22 \mathrm{a}$ & 34,4 & $13 \mathrm{a}$ & 35,1 \\
Hiperecogênicidade & $31 \mathrm{a}$ & 48,4 & $21 \mathrm{a}$ & 56,8 \\
\hline Total & 59 & 100 & 37 & 100 \\
\hline
\end{tabular}

Médias seguidas pela mesma letra na coluna não diferem estatisticamente entre si $(\mathrm{P}>0,05)$.

Do total de animais $(\mathrm{n}=70), 55$ animais possuíam alterações na avaliação ultrassonográfica do baço, sendo que $49,1 \%$ deles apresentaram esplenomegalia, mas a ecotextura do parênquima esplênico e ecogênicidade foram preservadas, não havendo diferença estatística com outras alterações ultrassonográficas sugestivas de linfoma esplênico (Tabela 3). Oito cães foram submetidos ao exame citológico/ histológico, em um animal foi realizada a análise histopatológica de amostra coletada durante a necropsia. As alterações ultrassonográficas dos animais com diagnóstico definitivo de infiltração neoplásica em baço demonstraram-se variáveis. Três cães apresentaram uma única massa de ecogenicidade mista e contornos definidos (Figura 3), um cão com massa cavitária de contornos definidos e quatro cães com lesões hipoecogênicas no parênquima esplênico, sendo focal em um cão e multifocal nos outros três. Mesmo na ausência de alterações nas imagens ultrassonográficas, um dos animais apresentou amostras citológicas compatíveis com linfoma esplênico.

Tabela 3. Distribuição das alterações ultrassonográficas do parênquima esplênico dos animais diagnosticados com linfoma por citologia de linfonodo superficial.

\begin{tabular}{lccccc}
\hline $\begin{array}{l}\text { Aspectos } \\
\text { ultrassonográficos da }\end{array}$ & \multicolumn{2}{c}{ Animais } & \multicolumn{3}{c}{ Esplenomegalia } \\
ecotextura do parênquima & $\mathrm{N}$ & $\%$ & Presença & $\%$ \\
esplênico & & & & & \\
\hline Preservado & $30 \mathrm{a}$ & 54,5 & $12 \mathrm{a}$ & 44,4 \\
Lesões focais & $14 \mathrm{~b}$ & 25,4 & $07 \mathrm{a}$ & 26,0 \\
Hiperecogênicidade difusa & $06 \mathrm{~b}$ & 11,0 & $04 \mathrm{a}$ & 14,8 \\
Hipoecogênicidade difusa & $05 \mathrm{~b}$ & 9,1 & $04 \mathrm{a}$ & 14,8 \\
\hline Total & 55 & 100 & 27 & 100 \\
\hline
\end{tabular}

Médias seguidas pela mesma letra na coluna não diferem estatisticamente entre si $(\mathrm{P}>0,05)$.
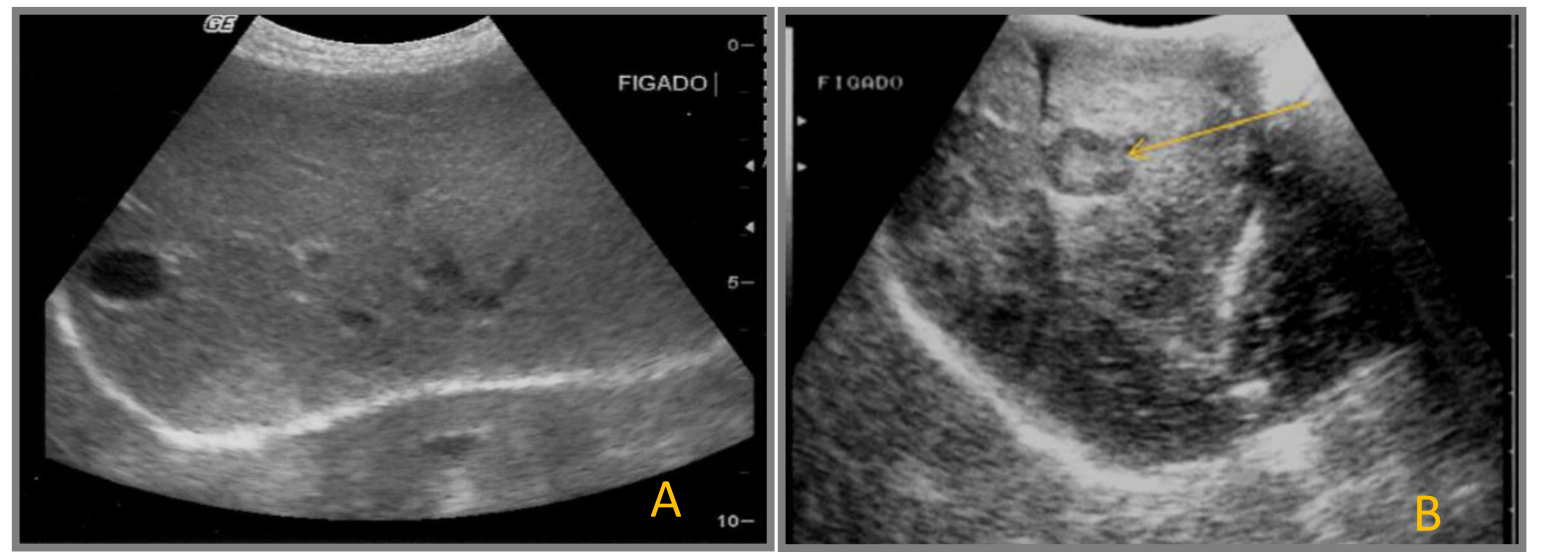

Figura 2. Imagem ultrassonográfica do parênquima hepático com diagnóstico citológico de linfoma (A) Aumento difuso da ecogenicidade de parênquima, associado à hepatomegalia. B) Lesão focal, do tipo “alvo” em parênquima hepático (seta). 

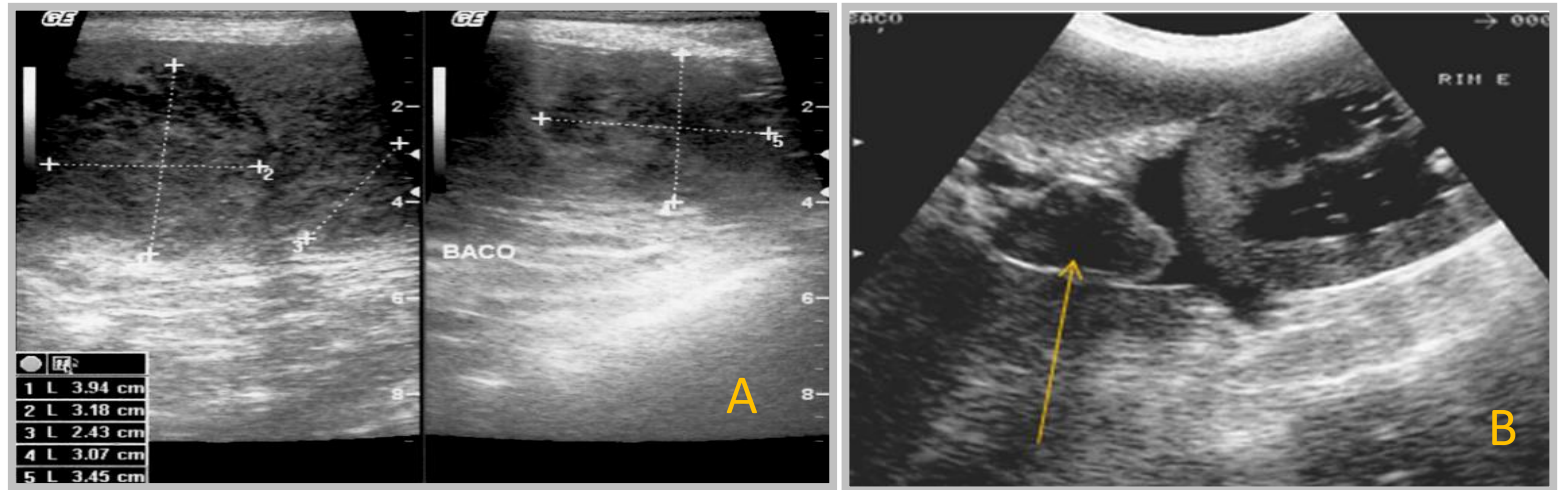

Figura 3. Imagem ultrassonográfica do parênquima esplênico de cães com diagnóstico citológico de linfoma. A) Lesão nodular de ecogenicidade mista em parênquima esplênico. B) Lesão cavitária esplênica compatível com linfoma (seta), presença de líquido livre caudal a lesão.

No estadiamento ultrassonográfico abdominal (Quadro 1) foi avaliado o grau de acometimento intra-abdominal e classificado segundo as alterações ultrassonográficas encontradas. Vinte animais dos 70 avaliados $(28,6 \%)$ foram classificados como estágio I; $1 / 70(1,4 \%)$ estágio II; 29/70 (41,4\%) estágio IV; $20 / 70(28,6 \%)$ estágio V. Neste estudo não foram classificados animais no estágio III.

Outro achado relevante foram as lesões hipoecogênicas de parênquima prostático com aumento de volume do órgão e diagnóstico citológico da próstata definitivo de linfoma.

Dos achados ultrassonográficos, três cães apresentaram espessamento superior a 7,0 $\mathrm{mm}$ em parede gástrica juntamente com decréscimo da ecogenicidade e perda da estratificação parietal, em que apenas um exibia lesão hipoecogênica focal e intramural em corpo de estômago, após a excisão cirúrgica foi comprovado na hitopatologia linfoma gástrico (Figura 4).

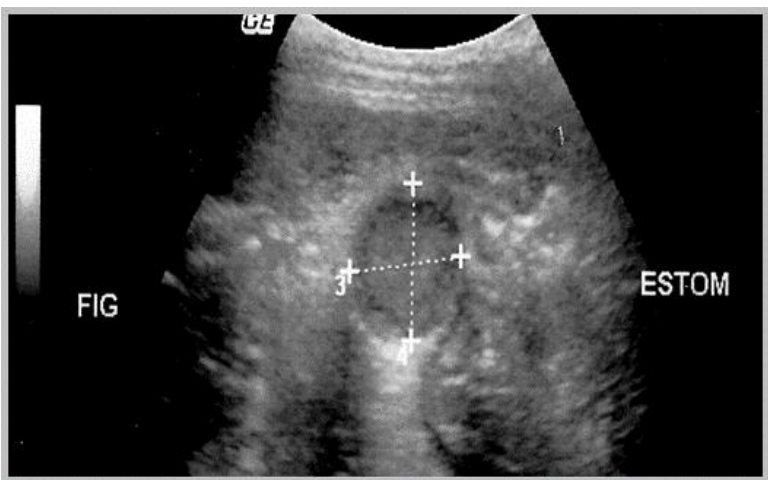

Figura 4. Imagem ultrassonográfica gástrica demonstrando lesão focal nodular, intramural, predominantemente hipoecogênica com perda da estratificação de camadas gástricas e diagnóstico definitivo de linfoma pela histopatologia.

\section{Discussão}

O linfoma canino é uma doença de apresentação clínica variável o que, na maioria das vezes dificulta um rápido diagnóstico. Conhecer as alterações ultrassonográficas desta doença auxilia na obtenção de diagnóstico precoce e contribui para o prognóstico. E segundo a análise estatística deste estudo, um órgão de dimensões normais não descarta o acometimento de linfoma nos cães.

A ocorrência de organomegalia em cães com linfoma é variável, como já descrita, em que autores encontraram 53\% de hepatomegalia e $46 \%$ de esplenomegalia (Blackwood et al. 1997). E em contrapartida um estudo recente observou que apenas $17,5 \%$ dos cães apresentavam aumento hepático e que 96,5\% apresentavam aumento esplênico (Gavazza et al. 2009). No presente estudo, a hepatomegalia esteve evidente em 54,9\% dos animais avaliados, a esplenomegalia em $49,1 \%$ e linfadenomegalia abdominal em $57,1 \%$. Entretanto, o aumento do tamanho destes órgãos pode estar relacionado à diversas causas como neoplasias concomitantes, hemoparasitoses, congestão, infecções bacterianas e abscessos, não sendo patognomônico de infiltrados neoplásicos (Ueno et al. 2009).

$\mathrm{O}$ viés do trabalho foi o fato de não realizar a punção de todos os órgãos abdominais alterados, pois os animais, na sua maioria, eram encaminhados ao exame ultrassonográfico após a confirmação de linfoma por aspiração de linfonodo superficial e por ser um estudo retrospectivo impossibilitou a confirmação de todas as alterações. Todavia, a medicina utiliza técnicas de imagem para o estadiamento de linfomas e acompanhamento da resposta 
terapêutica mesmo sem realizar a punção de todas as alterações (Borba et al. 2007).

A punção de todas as lesões é uma prática pouco rotineira no diagnóstico de linfomas multicêntricos, pois alguns linfonodos intraabdominais são de difícil acesso e geralmente estão adjacentes a grandes vasos, o que torna o procedimento mais arriscado devido a sangramentos. Como o diagnóstico geralmente é tardio alguns animais estão debilitados para serem anestesiados para punção de lesões abdominais profundas.

Dentre os aumentos das dimensões de linfonodos abdominais avaliados no estudo, detectou-se uma maior incidência do aumento dos linfonodos ilíacos mediais, podendo ser útil na triagem ultrassonográfica de cães com linfoma, visto que estudos prévios diferenciaram por meio do exame ultrassonográfico os linfonodos neoplásicos dos linfonodos normais (Llabrés-Díaz 2004). Neste estudo, os linfonodos que apresentam infiltrações neoplásicas possuem dimensões aumentadas, são arredondados e de ecogenicidade heterogênea à ultrassonografia (Llabrés-Díaz 2004).

Nos 59 cães com relatos de alterações do parênquima hepático, $82,8 \%$ foram difusas, dos 55 cães com avaliação esplênica $45,5 \%$ apresentaram alteração ultrassonográfica do baço, porém nem todos os animais possuíram avaliação citológica destes órgãos. A citologia aspirativa por agulha fina guiada pelo ultrassom poderia mudar o estadiamento clínico desses cães. A associação de exames ultrassonográficos e punção aspirativa guiada de lesões focais ou parenquimatosas proporcionam dados importantes e complementares sobre doenças e infiltrações neoplásicas, devendo essa técnica preceder procedimentos mais invasivos (Faverzani et al. 2006). Resultados anteriores ressaltam a importância da confirmação diagnóstica pela citologia, principalmente, em animais com aparência ultrassonográfica esplênica anormal para permitir a exclusão de processos benignos como, por exemplo, hematopoiese extramedular (Crabtree et al. 2010). Independentemente das alterações ultrassonográficas hepáticas encontradas deve-se realizar punção para determinação definitiva de linfoma (Crabtree et al. 2010), pois no presente estudo $7,8 \%$ dos fígados avaliados e $54,5 \%$ dos baços apresentaram-se de ecogênicidade normal e dimensões preservadas.
Ao se analisar o envolvimento dos linfonodos, o aumento dos ilíacos mediais foi significativamente mais frequente $(62,2 \%)$ do que o aumento dos demais linfonodos, sendo um dado relevante para triagem de linfoma canino.

Em humanos o acometimento prostático por linfomas primários ou secundários é infrequente, geralmente apresentando prognóstico desfavorável e sua apresentação ultrassonográfica é indistinguível de hiperplasia prostática benigna ou prostatite crônica (Zuazu et al. 2009). Em cães a literatura também é escassa sobre infiltrados neoplásicos prostáticos compatíveis com linfoma, segundo os resultados deste estudo deve-se incluir como diagnóstico diferencial de linfoma em cães as lesões hipoecogênicas em parênquima e aumento prostático.

Outras modalidades de imagem como a tomografia computadorizada ou a ressonância magnética podem ser utilizadas para comprovar a existência de lesões em vários órgãos abdominais causadas pelo linfoma (Arango \& Alzate 2002; Yasuda et al. 2004); porém, essas modalidades de imagem são menos acessíveis que o exame ultrassonográfico.

\section{Conclusão}

Os resultados obtidos por meio da avaliação ultrassonográfica abdominal permitem concluir que o estadiamento ultrassonográfico abdominal proposto é um recurso que pode ser utilizado para caracterizar as lesões intra-abdominais do linfoma canino, e que o estágio IV foi o mais frequente.

A grande variação do aspecto ultrassonográfico dessas lesões nos órgãos linfoides dificulta a obtenção de um diagnóstico definitivo apenas pelas imagens ultrassonográficas, porém o acometimento dos linfonodos ilíacos mediais pode ser sinal sentinela importante na suspeita de linfoma.

Os parênquimas prostáticos com aumento de volume e lesões hipoecogênicas deve incluir o linfoma no diagnóstico diferencial.

A inexistência de alteração em ecogenicidade e ecotextura dos parênquimas hepático e esplênico e a ausência de linfonodomegalia não descartam a presença de infiltrados neoplásicos nestes órgãos. Portanto, a ultrassonografia abdominal é um exame essencial para observar alterações que subsidiem a suspeita de linfoma canino, estadiamento clínico, prognóstico e monitorização da terapêutica instituída. 


\section{Referências bibliográficas}

Arango L.J.T. \& Alzate G. 2002. Linfosarcoma mediastínico en un perro de raza pitbull. Revista Colombiana de Ciencias Pecuarias 15, 315-318.

Blackwood L., Sullivan M. \& Lawson H. 1997. Radiographic abnormalities in canine multicentric lymphoma: a review of 84 cases. Journal of Small Animal Practice 38, 62-69.

Borba A.M.V., Monteiro A.M.V., Lima C.M.A.O., Ribeiro E.B., Lucena S.B.G. \& Skinner L.F. 2007. Aspectos da tomografia computadorizada no linfoma em pacientes abaixo de 20 anos de idade. Radiologia Brasileira 40, 87-92.

Crabtree A.C., Spangler E., Beard D. \& Smith A. 2010. Diagnostic accuracy of gray-scale ultrasonography for the detection of hepatic and splenic lymphoma in dogs. Veterinary Radiology \& Ultrasound 51, 661-664.

Dickinson R.M. 2008. Canine lymphosarcoma: Overcoming diagnostic obstacles and introduction to the latest diagnostic techniques. The Canadian Veterinary Journal 49, 305-308.

Faverzani S., Chinosi S., Valenti P. \& Caniatti M. 2006. Comparison between ultrasonography and cytology of liver focal lesions and parenchyma in the dog and cat. Veterinary Research Communications 30, 293-296.

Gavazza A., Sacchini F., Lubas G., Gugliucci B. \& Valori E. 2009. Clinical, laboratory, diagnostic and prognostic aspects of canine lymphoma: a retrospective study. Comparative Clinical Pathology 18, 291-299.

Jacobs R.M., Messick J.B. \& Valli V.E. 2002. Tumors of the hemolymphatic system. In: Tumors in Domestic Animals (ed. by Meuten DJ). Iowa State Press, Iowa, United States.

Llabrés-Díaz F.J. 2004. Ultrasonography of the medial iliac lymph nodes in the dog. Veterinary Radiology \& Ultrasound 45, 156-165.

Nyman H.T., Kristensen A.T., Skovgaard I.M. \& McEvoy F.J. 2005. Characterization of normal and abnormal canine superficial lymph nodes using gray-scale B-mode, color flow mapping, power, and spectral Doppler ultrasonography: a multivariate study. Veterinary Radiology \& Ultrasound 46, 404-410.

Ueno T.E.H., Aguiar D.M., Pacheco R.d.C., Rrichtzenhain L.J., Ribeiro M.G., Paes A.C., Megid J. \& Labruna M.B. 2009. Ehrlichia canis em cães atendidos em hospital veterinário de Botucatu, Estado de São Paulo, Brasil. Revista Brasileira de Parasitologia Veterinária 18, 57-61.

Vail D.M. 2000. Hematopoietic tumors. In: Textbook of Veterinary Internal Medicine (eds. by Ettinger SJ \& Feldman EC). Saunders, Philadelphia, United States.

Vail D.M. \& Young K.M. 2007. Hematopoietic Tumors. In: Small animal clinical oncology (eds. by Withrow SJ \& MacEwen EG). Elsevier, St. Louis, United States.

Wisner E.R. \& Pollard R.E. 2004. Trends in veterinary cancer imaging. Veterinary and Comparative Oncology 2, 49-74.

Yasuda D., Fujita M., Yasuda S., Taniguchi A., Miura H., Hasegawa D. \& Orima H. 2004. Usefulness of MRI compared with CT for diagnosis of mesenteric lymphoma in a dog. Journal of Veterinary Medical Science 66, 1447-1451.

Zuazu J.R., Iglesias R., Costa D.R., Mayans A.R., Roselló X.B., Santos Á.P. \& Polo J.M.B. 2009. Linfoma prostático y revisión de la literatura. Actas Urológicas Españolas 33, 686-690.

Recebido: 2 outubro, 2018.

Aprovado: 24 outubro, 2018.

Publicado: 26 dezembro, 2018.

Licenciamento: Este artigo é publicado na modalidade Acesso Aberto sob a licença Creative Commons Atribuição 4.0 (CC-BY 4.0), a qual permite uso irrestrito, distribuição, reprodução em qualquer meio, desde que o autor e a fonte sejam devidamente creditados. 